\title{
Improved Testing of the Magnetic-Field Integral Equation
}

\author{
Özgür Ergül, Student Member, IEEE, and Levent Gürel, Senior Member, IEEE
}

\begin{abstract}
An improved implementation of the magnetic-field integral equation (MFIE) is presented in order to eliminate some of the restrictions on the testing integral due to the singularities. Galerkin solution of the MFIE by the method of moments employing piecewise linear Rao-Wilton-Glisson basis and testing functions on planar triangulations of arbitrary surfaces is considered. In addition to demonstrating the ability to sample the testing integrals on the singular edges, a key integral is rederived not only to obtain accurate results, but to manifest the implicit solid-angle dependence of the MFIE as well.
\end{abstract}

Index Terms-Integral equations (IEs), magnetic-field integral equation (MFIE), moment methods, numerical analysis.

\section{INTRODUCTION}

A MULTITUDE of microwave applications have been enjoying the modeling and simulation capabilities offered by the recent progress in computational electromagnetics, especially by novel numerical methods employing iterative solvers and achieving fast matrix-vector multiplications, such as the fast multipole method (FMM) [1] and the multilevel fast multipole algorithm (MLFMA) [2], [3]. Even though the earlier implementations of these fast iterative solution methods employed the electric-field integral equation (EFIE) exclusively, the need to reduce the number of iterations for large problems necessitated the use of the combined-field integral equation (CFIE) [4], which involves the magnetic-field integral equation (MFIE) [5] in addition to the EFIE. Thus, the desire to reduce the iteration counts in a class of novel solvers created a renewed interest in the MFIE [3] despite its continuing importance that was recognized more than three decades ago [6].

In this letter, we consider the implementation of the MFIE within the context of versatile computational methods, such as the method of moments (MOM) [7], FMM, and MLFMA, which can treat piecewise planar surface triangulations of three-dimensional (3-D) arbitrary geometries. The unknown surface current is discretized with the Rao-Wilton-Glisson (RWG) [8] basis functions to obtain a piecewise linear approximation. RWG functions are also selected as testing functions in a Galerkin scheme. Then, the electromagnetic interactions of pairs of half RWG functions need to be computed via numerical integrations on both the basis and the testing triangles. However, the magnetic

Manuscript received May 5, 2005; revised May 20, 2005. This work was supported by the Turkish Academy of Sciences in the framework of the Young Scientist Award Program (LG/TUBA-GEBIP/2002-1-12), by the Scientific and Technical Research Council of Turkey (TUBITAK) under Research Grant 103E008, and by Contracts from ASELSAN and SSM.

The authors are with the Department of Electrical and Electronics Engineering, Bilkent University, Ankara R-06800, Turkey (e-mail: ergul@ee. bilkent.edu.tr; lgurel@bilkent.edu.tr).

Digital Object Identifier 10.1109/LMWC.2005.856697

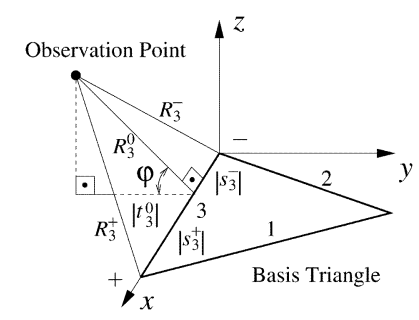

Fig. 1. Definition of the geometric variables for the near-neighbor interactions, where the observation point is a sampling point on the testing triangle.

field is usually singular at the edges of a basis triangle. This leads to numerical difficulties, especially for the near-neighbor interactions, for which the basis and testing triangles are touching. The difficulties have been traditionally circumvented in the literature by choosing the integration points strictly inside the testing triangle, i.e., by avoiding to sample the singularity at the edge. In this letter, we report for the first time, to our knowledge, an implementation of the MFIE achieving the sampling of the integrals on the edges of the basis and testing triangles, even for the singular near-neighbor interactions.

\section{MFIE FORMULATION}

Among various MFIE formulations [9] that are suitable for 3-D MOM implementations employing the RWG basis and testing functions, the most widely used formulation expresses the elements of the impedance matrix as

$$
\begin{aligned}
Z_{m n}= & \int_{S_{m}} d \boldsymbol{r t}_{m}(\boldsymbol{r}) \cdot \boldsymbol{b}_{n}(\boldsymbol{r}) \\
& -\int_{S_{m}} d \boldsymbol{r t _ { m }}(\boldsymbol{r}) \cdot \hat{\boldsymbol{n}} \times \int_{S_{n}} d \boldsymbol{r}^{\prime} \boldsymbol{b}_{n}\left(\boldsymbol{r}^{\prime}\right) \times \nabla^{\prime} g\left(\boldsymbol{r}, \boldsymbol{r}^{\prime}\right)
\end{aligned}
$$

where $\boldsymbol{t}_{m}$ represents the $m$ th testing function, $\boldsymbol{b}_{n}$ represents the $n$th basis function, and

$$
g\left(\boldsymbol{r}, \boldsymbol{r}^{\prime}\right)=\frac{e^{i k R}}{4 \pi R}, \quad R=\left|\boldsymbol{r}-\boldsymbol{r}^{\prime}\right|
$$

denotes the free-space Green's function in phasor notation with $e^{-i w t}$ convention. Due to the singularity of the MFIE kernel, accurate and efficient computation of (1) requires the use of the singularity-extraction methods for both the inner integral [10] and the outer integral [9], [11] especially for the near-neighbor interactions. Without losing generality, the basis triangle can be rotated to lie on the $x-y$ plane and to align one of its edges along the $x$ axis, as shown in Fig. 1. Then, extracting the singularity of the inner integral of (1) calls for the analytical evaluation of the three basic integrals

$$
I_{1}=\int_{S_{n}} d \boldsymbol{r}^{\prime} \frac{\left(x-x^{\prime}\right)}{R^{3}}
$$




$$
\begin{aligned}
I_{2} & =\int_{S_{n}} d \boldsymbol{r}^{\prime} \frac{\left(y-y^{\prime}\right)}{R^{3}} \\
I_{3} & =\int_{S_{n}} d \boldsymbol{r}^{\prime} \frac{z}{R^{3}}
\end{aligned}
$$

over the basis triangles. Analytical expressions for these three integrals are derived in [12], however, the derivation of $I_{3}$ in (3c) will be revisited in Section III.

As the observation point in Fig. 1 approaches the edge of the basis function, $I_{1}$ and $I_{2}$ become singular. Since the triangulation of an arbitrary geometry gives rise to many such edges, all near-neighbor interactions, where the basis and testing triangles share a common edge, involve an outer integral with a singular integrand. For this reason, earlier implementations of the MFIE opted for sampling this singular integrand strictly inside the testing triangle, hence avoiding the singular edges. A rare example of sampling at the edges [13] employs a non-Galerkin scheme, where the testing is performed at a constrained direction in order to avoid the singularity. We propose to improve the testing of the MFIE by removing all of the restrictions over the choice of the sampling points in the testing triangle. This can be achieved by employing a novel scheme to extract the logarithmic singularities in the outer integral [9], [11]. Such a scheme enhances not only the versatility of the testing procedure by allowing sampling at the edges, but also the accuracy and the efficiency of the numerical integrations, as demonstrated in [9].

As the testing point approaches the edge of the basis triangle, the logarithmic singularities in the outer integral caused by the inner integrals similar to $I_{1}$ and $I_{2}$ in (3a) and (3b) can be handled according to [9]. For the same limit case, the value of the remaining integral $I_{3}$ in (3c), although not singular, should be correctly determined. For this purpose, $I_{3}$ will be rederived in Section III.

\section{DERIVATION OF $I_{3}$}

When the basis triangle is on the $x-y$ plane as depicted in Fig. 1, the value of the third integral in (3c) is given as

$$
I_{3}=\int_{S_{n}} d \boldsymbol{r}^{\prime} \frac{z}{R^{3}}=\operatorname{sgn}(z) \beta
$$

where

$$
\beta=\sum_{i=1}^{3} \beta_{i}
$$

and

$$
\beta_{i}=\tan ^{-1} \frac{t_{i}^{0} s_{i}^{+}}{\left(R_{i}^{0}\right)^{2}+|z| R_{i}^{+}}-\tan ^{-1} \frac{t_{i}^{0} s_{i}^{-}}{\left(R_{i}^{0}\right)^{2}+|z| R_{i}^{-}} .
$$

The geometric variables in (6) are shown in Fig. 1 for the edge, which the observation point approaches $(i=3)$. As explained in [12], some of the variables have signs that are defined as

$$
t_{3}^{0}=-\left|t_{3}^{0}\right| \quad s_{3}^{-}=-\left|s_{3}^{-}\right| \quad s_{3}^{+}=\left|s_{3}^{+}\right| \text {. }
$$

Then, as the observation point approaches the edge

$$
\begin{aligned}
\lim _{R_{3}^{0} \rightarrow 0} \beta_{3} & =-\operatorname{sgn}(z)\left\{\tan ^{-1} \frac{\cos (\varphi)}{\sin (\varphi)}+\tan ^{-1} \frac{\cos (\varphi)}{\sin (\varphi)}\right\} \\
& =2 \operatorname{sgn}(z) \varphi-\pi
\end{aligned}
$$

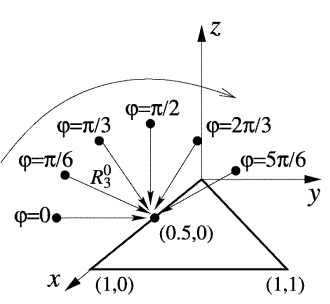

(a)

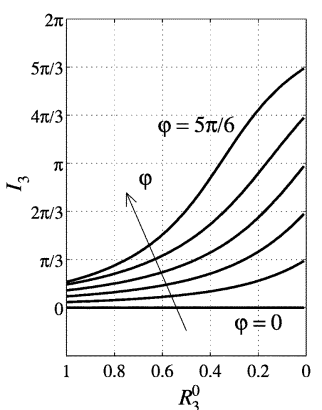

(b)
Fig. 2. (a) Observation points approaching the edge of the basis triangle at different angles. (b) The value of $I_{3}$ in (3c) for various approach angles of the observation point.

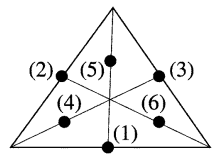

(a)

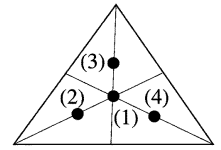

(b)

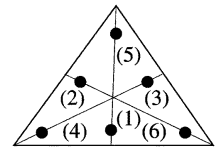

(c)
Fig. 3. Sampling points used for the numerical integration on the testing triangle.

where $\varphi$ is the angle between the approach path of the observation point and the $x-y$ plane, and

$$
\lim _{R_{3}^{0} \rightarrow 0}\left(\beta_{1}+\beta_{2}\right)=\pi
$$

so that

$$
\lim _{R_{3}^{0} \rightarrow 0} \int_{S_{n}} d \boldsymbol{r}^{\prime} \frac{z}{R^{3}}=\operatorname{sgn}(z) \operatorname{sgn}(z) 2 \varphi=2 \varphi .
$$

Consequently, in the calculation of the interactions between touching basis and testing triangles, the value of $I_{3}$ depends on the angle between the triangles when the observation point approaches the edge. In [12], the limit value is given as

$$
\text { “ } \beta_{i}=0 \text { if } t_{i}^{0}=0 \quad \forall z "
$$

which is correct only for the approaches along the $z$ axis, i.e., $\varphi= \pm \pi / 2$.

Fig. 2(b) presents the value of $I_{3}$ with respect to the distance between the observation point and the edge of the basis triangle $\left(R_{3}^{0}\right)$ for different approach angles as depicted in Fig. 2(a). The curves in Fig. 2(b) clearly indicate that the value of $I_{3}$ depends on the angle of approach, which is the same as the angle between the testing and basis triangles. This is also related to the solid angle of the wedge formed by the basis and testing triangles. Indeed, this is exactly how the solid-angle factor of the MFIE can be computed correctly and implicitly [11], [14].

\section{RESULTS}

To demonstrate the necessity of the correct calculation of the limit value in (10), we present the results of a scattering problem involving a perfectly conducting sphere of radius $r_{0}=0.3 \lambda$. Fig. 3(a) shows the sampling points on the testing triangles used for the numerical integrations. Using the six-point integration rule illustrated in Fig. 3(a), which is merely an example (any other numerical integration scheme can also be employed), the result of the integration over a triangle is expressed as [15]

$$
\int_{S_{m}} f(\boldsymbol{r}) d \boldsymbol{r}=\frac{A_{m}}{30}\left\{\sum_{i=1}^{3} f_{i}+9 \sum_{i=4}^{6} f_{i}\right\}
$$




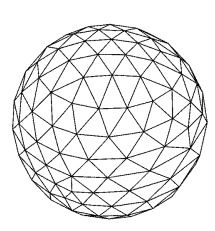

(a)

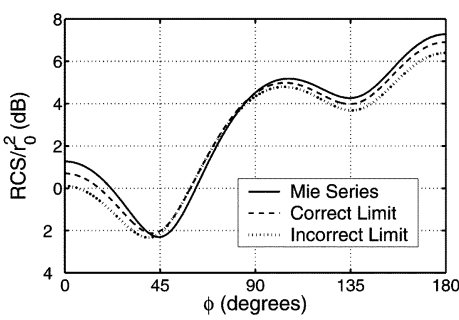

(b)
Fig. 4. (a) Conducting sphere of radius $0.3 \lambda$ with $\lambda / 10$ triangulation. (b) Total value of normalized RCS (RCS/ $r_{0}^{2}$ in decibels) on the $x-y$ plane for a sphere illuminated by a $y$-polarized plane wave propagating in the $-x$ direction.

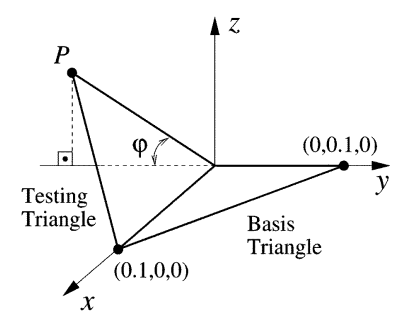

Fig. 5. Geometric configuration of two touching triangles.

where $A_{m}$ is the area of the triangle $S_{m}$ and $f_{i}$ is the value of the integrand at point $i=1,2, \ldots, 6$. The first three points are located on the edges of the testing triangle, and therefore, they are also on the edges of the neighboring basis triangles.

Fig. 4(a) shows the geometry with $\lambda / 10$ triangulation and Fig. 4(b) shows the total value of the normalized radar cross section $\left(\mathrm{RCS} / r_{0}^{2}\right.$ in $\left.\mathrm{dB}\right)$ on the $x-y$ plane when the sphere is illuminated by a $y$-polarized plane wave with incidence in the $-x$ direction. Mie series result is compared to the numerical results employing (10) (correct limit) and (11) (incorrect limit). We have confirmed that the small discrepancy between the Mie series and "correct" numerical results is due to the coarse meshing of the sphere, i.e., this small error disappears as the mesh becomes finer. On the other hand, the incorrect use of the limit value as given in (11) causes a persistent error that cannot be corrected by refining the mesh. This example demonstrates the successful implementation of the testing of the MFIE on the triangle edges and that it is critical to correctly evaluate the limit value $I_{3}$ in (3c) to obtain accurate results.

Furthermore, Table I shows that electromagnetic interactions between pairs of touching triangles are computed more accurately by sampling the testing integral on the edges compared to sampling strictly inside the testing triangle. As opposed to the previous paragraph, incorrect limit of the integral is not used in any of the three cases. Fig. 5 depicts the interaction of two touching triangles with an angle $180^{\circ}-\varphi$ between them. The reference values, which are used to assess the percent error of other results in Table I, are obtained in [9] by using higher-order integrations. The six-point numerical integration rule [Fig. 3(a)] sampling the testing integral on the edges performs better than the four-point rule [Fig. 3(b)] sampling strictly inside the testing triangle. Both integration rules are of third order [16]. Testing on the edges [Fig. 3(a)] performs even better than the six-point integration rule in [Fig. 3(c)], which is of the fourth order [16]. All errors can be further reduced by adaptively dividing the testing triangle.
TABLE I

Electromagnetic INTERACTIONS BETWEEN PAIRS OF Two TOUCHING TRIANGLES

\begin{tabular}{ccc}
$\begin{array}{c}\text { Angle between the triangles } \\
\left(180^{\circ}-\varphi\right)\end{array}$ & $150^{\circ}$ & $120^{\circ}$ \\
\hline Reference values [9] & $3.055 \mathrm{e}-2$ & $6.288 \mathrm{e}-2$ \\
\hline 4-point integration & $2.634 \mathrm{e}-2$ & $5.486 \mathrm{e}-2$ \\
strictly inside & (Error: 13.8\%) & (Error: 12.8\%) \\
\hline $\begin{array}{c}\text { 6-point integration } \\
\text { strictly inside }\end{array}$ & $2.788 \mathrm{e}-2$ & $5.799 \mathrm{e}-2$ \\
(Error: $8.7 \%)$ & (Error: 7.8\%) \\
\hline 6-point integration & $3.185 \mathrm{e}-2$ & $6.466 \mathrm{e}-2$ \\
on the edges & (Error: $4.3 \%)$ & (Error: 2.8\%) \\
\hline
\end{tabular}

\section{CONCLUSION}

In this letter, we report an improvement for the testing of the MFIE leading to the freedom to sample on the edges of the testing triangle, even for the singular near-neighbor interactions. This improvement, which allows for the Galerkin implementations of the MFIE solutions with RWG discretizations, requires the use of a novel singularity-extraction method [9], [11]. Testing on the edges of the triangular domains relies on the correct calculation of the limit values of some integrals. One such critically important integral is rederived in this letter not only to obtain a correct limit value, but also to establish the implicit solid-angle dependence of the MFIE [11], [14].

\section{REFERENCES}

[1] R. Coifman, V. Rokhlin, and S. Wandzura, "The fast multipole method for the wave equation: A pedestrian prescription," IEEE Antennas Propag. Mag., vol. 35, no. 3, pp. 7-12, Jun. 1993.

[2] C.-C. Lu and W. C. Chew, "Multilevel fast multipole algorithm for electromagnetic scattering by large complex objects," IEEE Trans. Antennas Propag., vol. 45, no. 10, pp. 1488-1493, Oct. 1997.

[3] W. C. Chew, J.-M. Jin, E. Michielssen, and J. Song, Fast and Efficient Algorithms in Computational Electromagnetics. Boston, MA: Artech House, 2001.

[4] J. R. Mautz and R. F. Harrington, "H-field, E-field, and combined field solutions for conducting bodies of revolution," $A E \ddot{U}$, vol. 32, no. 4, pp. 157-164, Apr. 1978.

[5] N. Morita, N. Kumagai, and J. R. Mautz, Integral Equation Methods for Electromagnetics. Boston, MA: Artech House, 1990.

[6] A. J. Poggio and E. K. Miller, "Integral equation solutions of three-dimensional scattering problems," in Computer Techniques for Electromagnetics, R. Mittra, Ed. New York: Permagon, 1973, ch. 4.

[7] R. F. Harrington, Field Computation by Moment Methods. New York: Macmillan, 1968.

[8] S. M. Rao, D. R. Wilton, and A. W. Glisson, "Electromagnetic scattering by surfaces of arbitrary shape," IEEE Trans. Antennas Propagat., vol. AP-30, no. 5, pp. 409-418, May 1982.

[9] L. Gürel and Ö. Ergül, "Singularity of the magnetic-field integral equation and its extraction," IEEE Antennas Wireless Propag. Lett., vol. 4 , pp. 229-232, 2005.

[10] R. E. Hodges and Y. Rahmat-Samii, "The evaluation of MFIE integrals with the use of vector triangle basis functions," Microw. Opt. Technol. Lett., vol. 14, no. 1, pp. 9-14, Jan. 1997.

[11] Ö. Ergül and L. Gürel, "Investigation of the inaccuracy of the MFIE discretized with the RWG basis functions," in Proc. IEEE AP-S Int. Symp. URSI Radio Sci. Meeting, Monterey, CA, Jun. 2004, pp. 3393-3396.

[12] R. D. Graglia, "On the numerical integration of the linear shape functions times the 3-D Green's function or its gradient on a plane triangle," IEEE Trans. Antennas Propag., vol. 41, no. 10, pp. 1448-1455, Oct. 1993.

[13] A. F. Peterson, S. L. Ray, and R. Mittra, Computational Methods for Electromagnetics. New York: IEEE Press, 1998.

[14] Ö. Ergül and L. Gürel, "Solid-angle factor in the magnetic-field integral equation," Microw. Opt. Technol. Lett., vol. 45, no. 5, pp. 452-456, Jun. 2005.

[15] A. H. Stroud, Approximate Calculation of Multiple Integrals. Englewood Cliffs, NJ: Prentice-Hall, 1971.

[16] D. A. Dunavant, "High degree efficient symmetrical gaussian quadrature rules for the triangle," Int. J. Numer. Meth. Eng., vol. 21, pp. 1129-1148, 1985. 